\title{
Combined 3D Chemical and Morphological Characterization Using Innovative Lab- based Equipment
}

\author{
Matthieu N. Boone $^{1, *}$, Brecht Laforce ${ }^{2}$, Bert Masschaele ${ }^{1,3}$, Manuel Dierick ${ }^{1}$, Frederic Van Assche ${ }^{1}$, \\ Veerle Cnudde $^{4}$, Bart Vekemans ${ }^{2}$, Laszlo Vincze ${ }^{2}$ and Luc Van Hoorebeke ${ }^{1}$ \\ 1. UGCT-RP, Dept. Physics and Astronomy, Ghent University, Proeftuinstraat 86/N12, B-9000 Gent, \\ Belgium \\ 2. XMI, Dept. Chemistry, Ghent University, Krijgslaan 281/S12, B-9000 Gent, Belgium \\ 3. XRE N.V., Bollebergen 2B/1, 9052 Zwijnaarde, Belgium \\ ${ }^{4}$ UGCT-PProGRess, Dept. Geology, Ghent University, Krijgslaan 281/S8, B-9000 Gent, Belgium \\ * Corresponding author, Matthieu.Boone@UGent.be
}

High-resolution X-ray computed tomography $(\mu \mathrm{CT})$ is nowadays an established technique for material characterization. However, the technique only yields morphological information, and the composition of the object can only be retrieved based on an educated guess. On the other hand, three-dimensional highresolution X-ray fluorescence spectroscopy $(\mu \mathrm{XRF})$ is an analytical technique that yields the chemical composition of a sample. In the past, several efforts have been made to combine both methods. Typically, they apply both techniques at different setups, and register the results [1,2]. One notable system developed earlier combines micro-CT and full-field XRF in one apparatus [3].

At the Ghent University, the X-ray Microspectroscopy and Imaging (XMI) research group and the Radiation Physics research group (RP, part of the Centre of X-ray Tomography UGCT) focus on combining chemical and morphological characterization of objects. On one hand, this is realized by the construction of a laboratory scanner system called Herakles that combines three measurement stages: one high-resolution transmission tomography stage, one confocal $\mu \mathrm{XRF}$ stage and one $\mu \mathrm{XRF}-\mathrm{CT}$ stage. This system allows for registration-free combination of morphological and chemical information at a high precision. On the other hand, an energy-dispersive X-ray detector providing both a high spatial and spectral resolution is used for hyperspectral X-ray transmission imaging combining both chemical and morphological characterization of objects simultaneously.

The Herakles system can be seen in Fig. 1 and is described in more detail in [4]. It is built on a large granite table and contains a high-precision air-bearing positioning system developed by LAB (Leuven, Belgium). This is required to move the sample between the different measurement stages, which corresponds to a maximum travel range of over $80 \mathrm{~cm}$. To avoid post-processing registration, the positioning accuracy needs to be better than $1 \mu \mathrm{m}$. The $\mu \mathrm{CT}$ stage is positioned in the middle. It consists of a transmission-type tube and two different detectors, one for low-attenuating samples and one for highattenuating and larger samples. The highest achievable resolution on this setup is approximately $500 \mathrm{~nm}$. The confocal XRF stage consists of a $50 \mathrm{~W}$ tube with Mo target equipped with polycapillary optics to generate a focused X-ray beam with a working distance of $3.6 \mathrm{~mm}$ and a spot size of $10 \mu \mathrm{m} \times 13 \mu \mathrm{m}$, and two large area SDD detectors, of which one is equipped with polycapillary optics. By aligning the focal points of both optics (i.e. tube and detector), a confocal measurement of a single microvoxel can be performed [4]. The size of this voxel is approximately $30 \times 30 \times 30 \mu \mathrm{m}^{3}$. The XRF-CT setup consists of a similar source with monocapillary optics, resulting in a pencil beam, and two large area SDD detectors, without optics, which are used to detect the spectrum emitted by the illuminated line in the sample. By stepping the sample sideways and rotating, a first-generation tomography setup is realized [5]. 


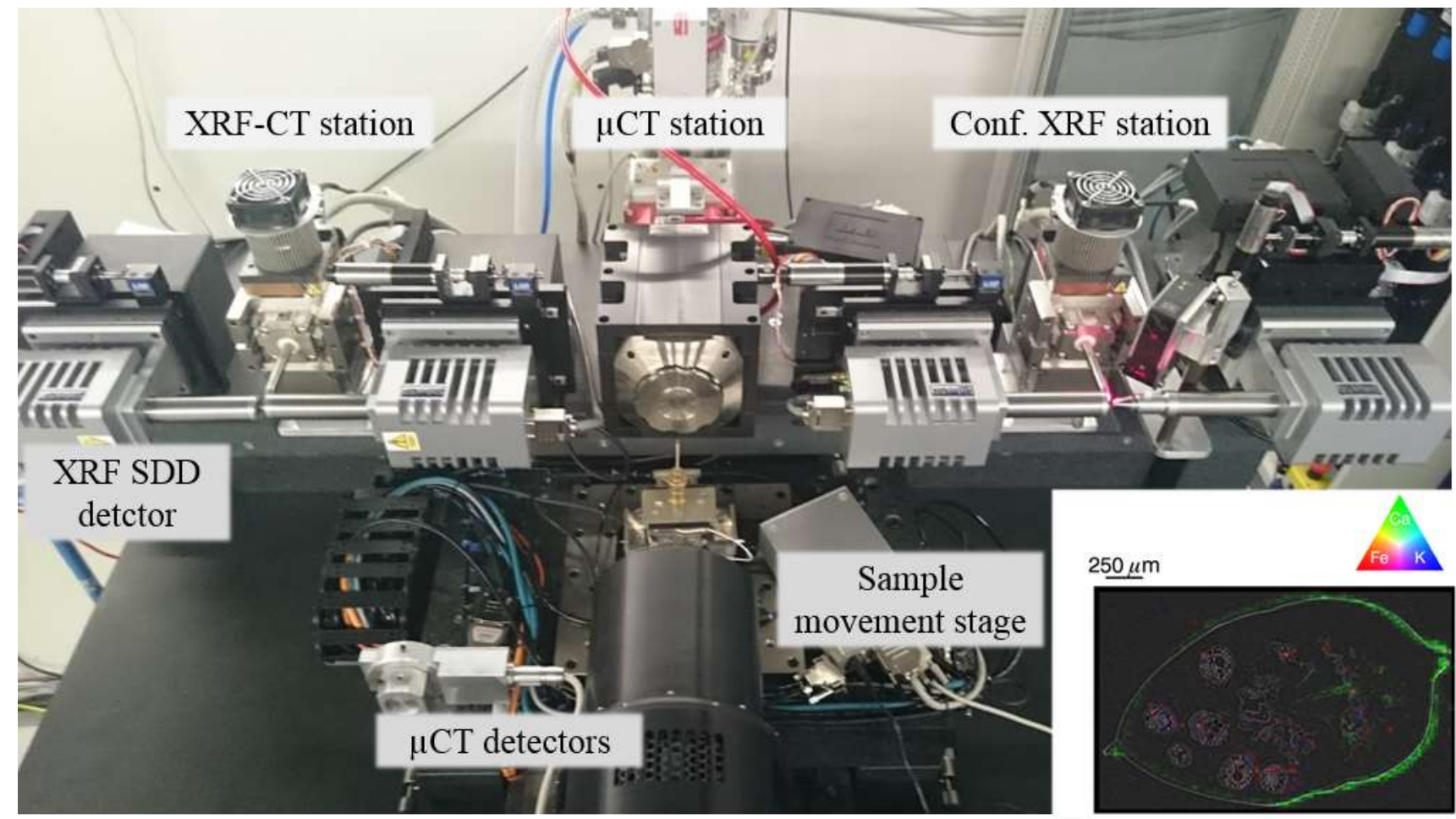

Figure 1. An overview of the Herakles scanner. The sample stage is located at the central $\mu \mathrm{CT}$ setup with a transmission-type X-ray tube and two different detectors. On the left side, the XRF-CT setup is equipped with two large-area SDDs and a tube with monocapillary optics. On the right side, the confocal $\mu \mathrm{XRF}$ setup is equipped with a tube with polycapillary optics, and two SDDs of which one is equipped with focusing optics. The inset shows an overlayed $\mu$ CT slice and XRF-CT scan of a daphnia magna [4]

The hyperspectral camera is described in more detail in [6]. Each pixel in the 264x264 matrix has a spectral resolution of approximately $160 \mathrm{eV}$, comparable with state-of-the-art single-pixel spectroscopic detectors. Although it is mostly used for X-ray fluorescence experiments, it can also be used for transmission imaging [7]. In this mode, the polychromatic nature of lab-based sources can be exploited by measuring the full attenuation spectrum for each pixel or voxel, giving information about the local chemical composition [8]. This methodology can be used for both radiography and tomography.

In this presentation, we describe both systems in more detail, and discuss the potential and the limitations of both techniques on a selection of illustrative examples. Particularly large attention will be given to murine aortic arches with atherosclerotic plaque and gold nanoparticle (AuNP) labelled monocytes [9]. This is an interesting use case. Whereas the calcium-rich plaque in the soft-tissue matrix makes grey valuebased localisation of the AuNP impossible, the concentration of gold is sufficient to be easily visualized on XRF images (Figure 2) and this application is highly relevant in (bio)medical sciences. These samples have been analysed at 1) the Heraklas scanner, 2) using hyperspectral imaging as well as 3) using L-edge imaging at the I13-2 imaging beamline at Diamond Light Source (Didcot, UK). This allows for crossmethod validation of the observed features. 

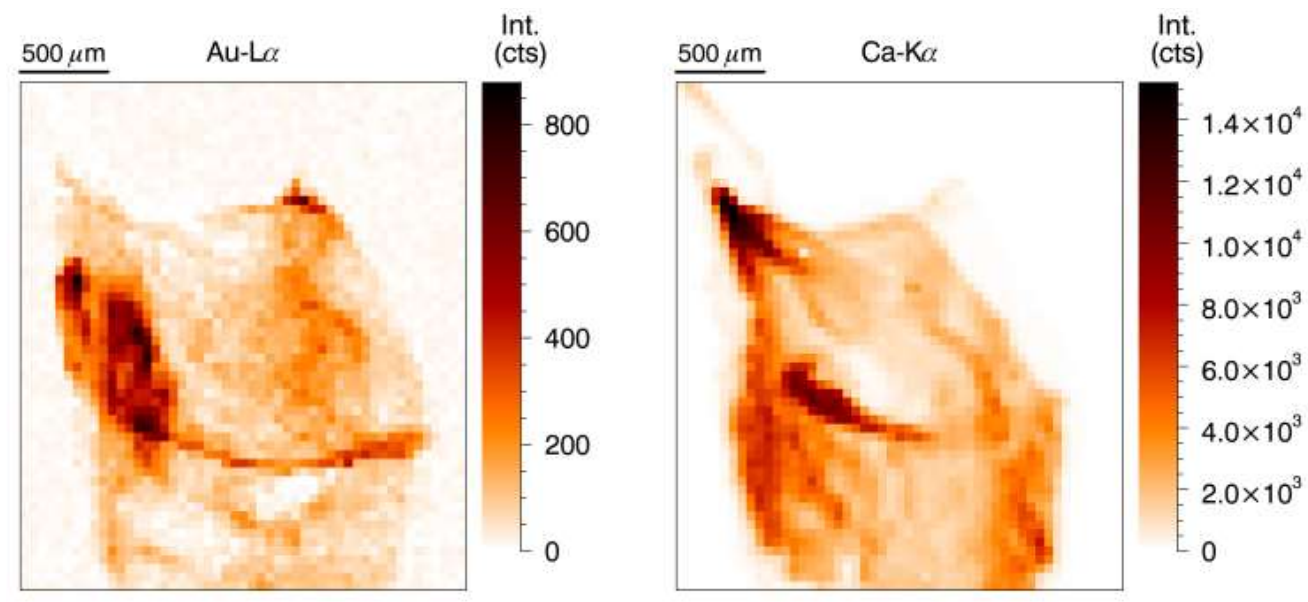

Figure 2. (Left and middle) XRF mappings of Au and Ca measured on the XRF-CT setup of the Herakles scanner. Given the measurement geometry, these mappings are integrated over the whole depth of the sample. (Right) Preliminary 3d mapping of Au measured with the SLcam hyperspectral detector [7].

[1] B De Samber et al., J. Anal. At. Spectrom. 24(4) (2010) p. 544-553.

[2] N L Cordes et al., Spectrochim. Acta Part B At. Spectrosc. 101 (2014), p. 320-329.

[3] P Bruyndonckx, A Sasov and X Liu, in XRM2010 Proceedings (2011) p.61-64..

[4] B Laforce et al, Anal. Chem. 89 (2017) 10617

[5] C G Schroer, Appl. Phys. Lett. 79(12) (2001), 1912-1914

[6] O Scharf et al., Anal. Chem. 83 (2011), 2532-2538

[7] M N Boone et al., Nucl. Instrum. Methods Phys. Res., Sect. A 735 (2014), p. 644-648

[8] C K Egan et al., Sci. Rep. 5 (2015) p. 15979

[9] P Chhour et al., Biomaterials 87 (2016) p. 93-103

[10] The setup was developed within the framework of a Hercules-II project (Project AUG11-024). The authors acknowledge funding from the Research Foundation - Flanders (FWO Research Project G0A0417N), the Special Research Fund of Ghent University and the IWT (Agentschap voor Innovatie door Wetenschap en Technology, Flanders) 
https://doi.org/10.1017/S1431927618013247 Published online by Cambridge University Press 